\title{
Intrinsic ferromagnetism versus phase segregation in Mn-doped Ge
}

\author{
E. Biegger, ${ }^{\text {a) }}$ L. Stäheli, M. Fonin, and U. Rüdiger \\ Fachbereich Physik, Universität Konstanz, 78457 Konstanz, Germany \\ Yu. S. Dedkov \\ Institut für Festkörperphysik, Technische Universität Dresden, 01062 Dresden, Germany
}

\begin{abstract}
We report on a detailed study of structural, magnetic, and electronic properties of $\mathrm{Mn}_{x} \mathrm{Ge}_{1-x}$ single crystals $(0<x<0.1)$ prepared by the Bridgman's crystal growth technique. The chemical distribution of $\mathrm{Mn}$ in a Ge matrix investigated by means of energy dispersive x-ray spectroscopy indicates a strong phase separation in the $\mathrm{Ge} / \mathrm{Mn}$ system. Temperature-dependent magnetization $[M(T)]$ measurements performed on the Mn-poor part containing 1\%-3\% of Mn reveal magnetic properties similar to those of a $\mathrm{Mn}_{11} \mathrm{Ge}_{8}$ reference sample as well as to those of the highly inhomogeneous Mn-rich parts of the sample. X-ray absorption spectroscopy (XAS) at the Mn $L_{2,3}$ absorption edge shows that $\mathrm{Mn}$ ions in Mn-rich and Mn-poor parts are in the divalent high-spin state. Resonant valence-band photoelectron spectroscopy (ResPES) was performed at the Mn $2 p_{3 / 2}$ absorption edge for the Mn-poor part. The obtained Mn $3 d$ PDOS shows a feature centered at 4.8 $\mathrm{eV}$ which is characteristic for the $\mathrm{Mn}_{5} \mathrm{Ge}_{3}$ phase. We identify the formation of ferromagnetic intermetallic compounds as the most possible origin of ferromagnetism in both Mn-rich and Mn-poor regions of the $\mathrm{Mn}_{x} \mathrm{Ge}_{1-x}$ samples.
\end{abstract}

\section{INTRODUCTION}

Diluted magnetic semiconductors (DMS) have become the subject of intensive research due to the possibility to utilize both charge and spin degrees of freedom in the same material which permits the design of a new generation of spintronic devices with enhanced functionalities. Recently, interest in DMS has been reinspired by the discovery of spontaneous ferromagnetism in $\mathrm{Mn}$-doped $\mathrm{GaAs}$ with a $\mathrm{Cu}-$ rie temperature $\left(T_{C}\right)$ of about $110 \mathrm{~K} .{ }^{1}$ Ferromagnetic ordering in the $\mathrm{Ga}_{1-x} \mathrm{Mn}_{x}$ As system can be understood on the basis of mean-field calculations of the Zener's model, ${ }^{2}$ which also predicts high $T_{C}$ ferromagnetism in several Mn-doped semiconducting materials. Recent experimental investigations revealed the presence of ferromagnetic order in $\mathrm{Mn}$ doped Ge thin films ${ }^{3,4}$ and in bulk single crystals ${ }^{5}$ showing $T_{C}$ of about 116 and $250 \mathrm{~K}$, respectively. Ferromagnetic order was also reported for epitaxial films of Ge codoped with $\mathrm{Mn}$ and Co with $T_{C}$ of about $270 \mathrm{~K}^{6}{ }^{6}$ Although much effort has been focused on the investigation of $\mathrm{Mn}_{x} \mathrm{Ge}_{1-x} \mathrm{DMS}$, the precise origin of the ferromagnetic order in this system is still unclear. In particular, the possibility of phase separation (formation of Mn-rich phases) in the Ge/Mn system and its influence on the magnetic properties of the DMS is the key issue in the field.

In the pioneering work by Cho et al., ${ }^{5} \mathrm{Mn}_{x} \mathrm{Ge}_{1-x}$ DMS single crystals were reported to show ferromagnetic order between 150 and $285 \mathrm{~K}$, as determined from temperaturedependent magnetization measurements. In this early study it was also suggested that $\mathrm{Mn}$ incorporates into the Ge host

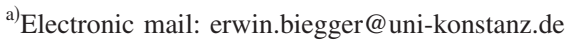

lattice without the formation of intermetallic phases, and the origin of the ferromagnetism in $\mathrm{Mn}_{x} \mathrm{Ge}_{1-x}$ is therefore intrinsic. Since then, various preparation techniques have been employed in order to produce Mn-doped Ge DMS, including single-crystal growth, ${ }^{5}$ molecular beam epitaxy (MBE) ${ }^{7-9}$ and ion implantation. ${ }^{10,11}$ However, several of the reports on intrinsic ferromagnetism in $\mathrm{Mn}_{x} \mathrm{Ge}_{1-x}$ DMS have been questioned recently by the structural proofs for the formation of intermetallic ferromagnetic compounds, both in single crystals $^{12}$ and low-temperature MBE fabricated films. Thus, an identification of phase separation in the Mn-doped Ge system would be helpful for the interpretation of the magnetic properties of $\mathrm{Mn}_{x} \mathrm{Ge}_{1-x}$.

In this article we report on the investigation of structural, magnetic, and electronic properties of $\mathrm{Mn}_{x} \mathrm{Ge}_{1-x}$ samples grown by the Bridgman's crystal growth technique. The chemical distribution of $\mathrm{Mn}$ in the Ge matrix investigated by means of energy dispersive x-ray spectroscopy (EDX) clearly shows a strong phase separation between Mn-rich and single-crystalline $\mathrm{Mn}$-poor regions. Temperature-dependent magnetization $[M(T)]$ measurements on Mn-poor parts of the $\mathrm{Mn}_{x} \mathrm{Ge}_{1-x}$ samples show ferromagnetic behavior between 150 and $285 \mathrm{~K}$, which was compared to that of a reference $\mathrm{Mn}_{11} \mathrm{Ge}_{8}$ sample. The electronic properties of the Mn-rich and -poor regions of the $\mathrm{Mn}_{x} \mathrm{Ge}_{1-x}$ sample were investigated by x-ray absorption spectroscopy (XAS) at $\mathrm{Mn} L_{2,3}$ absorption edge as well as valence-band photoemission spectroscopy. Resonant photoelectron spectroscopy (ResPES) was performed at the Mn $2 p-3 d$ excitation threshold to obtain the partial density of states (PDOS) of Mn $3 d$ states in the Mn-poor region. 

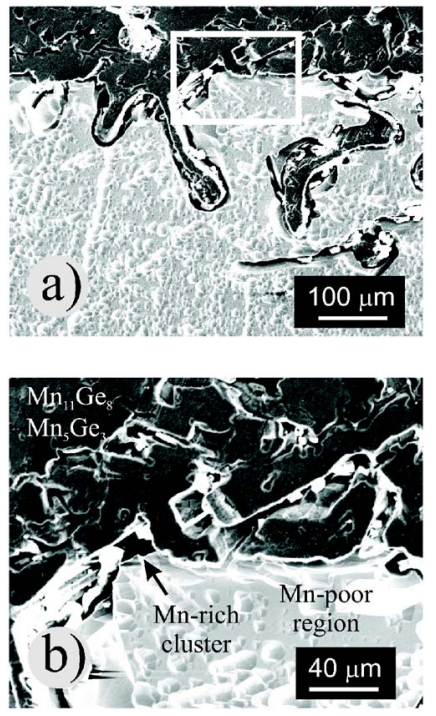

FIG. 1. (a) $640 \times 460 \mu \mathrm{m}^{2}$ SEM image of the $\mathrm{Mn}_{0.1} \mathrm{Ge}_{0.9}$ crystal surface with dark regions corresponding to the Mn-rich phase and bright regions corresponding to the Mn-poor phase. (b) $260 \times 180 \mu \mathrm{m}^{2}$ zoom into the field marked in (a). The chemical composition determined by EDX is marked in image (b)

\section{EXPERIMENT}

$\mathrm{Mn}_{x} \mathrm{Ge}_{1-x}$ single crystals were prepared by means of the Bridgman's crystal growth technique from high-purity $\mathrm{Mn}$ $(3 N)$ and $\mathrm{Ge}(5 N)$ powders. The overall sample preparation procedure used in this study was close to that of Cho et al. ${ }^{5}$ In brief, $\mathrm{Ge}$ and $\mathrm{Mn}$ powders were mixed and encapsulated in a quartz ampoule. The ampoule was heated to $1000{ }^{\circ} \mathrm{C}$ in a vertical furnace followed by a $48 \mathrm{~h}$ soak. The crystal growth was performed by decreasing the temperature at $0.5^{\circ} \mathrm{C} / \mathrm{h}$ below the melting point of $\mathrm{Ge}\left(937^{\circ} \mathrm{C}\right)$ to about $850{ }^{\circ} \mathrm{C}$ and thereafter at $100{ }^{\circ} \mathrm{C} / \mathrm{h}$. After the growth the single crystals were cut with a wire saw in order to isolate the distinct parts of the sample. XAS and ResPES experiments were carried out at the RGBL-PGM beam line of BESSY (Berlin). ResPES spectra were collected in the angleintegrated mode with a total energy resolution of $150 \mathrm{meV}$. The photoemission spectra were normalized to the incident photon flux, which was monitored by detecting the photocurrent from the last gold-covered mirror of the beam line. XAS spectra were obtained by employing the total electron yield method at the Mn $L_{2,3}$ absorption edge with the photonenergy resolution set to $80 \mathrm{meV}$. After introducing the sample into the UHV chamber at BESSY its surface was cleaned by moderate $\mathrm{Ar}^{+}$ion sputtering.

\section{RESULTS AND DISCUSSION}

\section{A. Structural properties}

EDX on a scanning electron microscope (SEM) was performed to investigate the chemical distribution of $\mathrm{Mn}$ in the samples. Figure 1 shows a SEM image $\left(640 \times 460 \mu \mathrm{m}^{2}\right)$ of the $\mathrm{Mn}_{0.1} \mathrm{Ge}_{0.9}$ crystal surface (a) together with a 260 $\times 180 \mu \mathrm{m}^{2}$ zoom (b) into the field marked in (a). Careful EDX analysis at the surface shows a strong phase separation between Mn-rich and Mn-poor regions. In Mn-poor regions

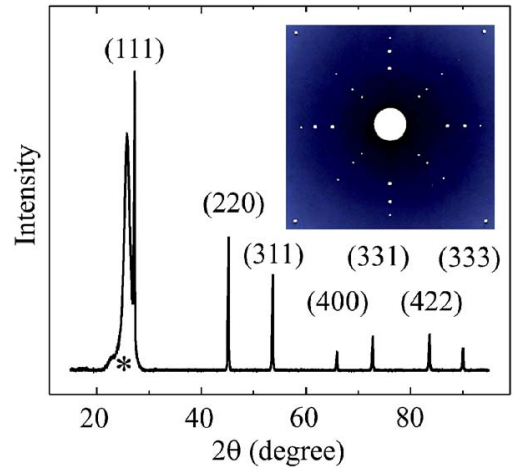

FIG. 2. $\theta-2 \theta$ x-ray powder diffraction pattern of a Mn-poor part of the $\mathrm{Mn}_{0.08} \mathrm{Ge}_{0.92}$ sample. The peak labeled with an asterisk is due to the sample holder. The inset shows a Laue pattern taken along the [100] direction of the single-crystal $\mathrm{Mn}_{0.08} \mathrm{Ge}_{0.92}$ sample, confirming the good crystalline quality of the Mn-poor region.

(bright areas in Fig. 1) the Mn content was found to be in the range of $1 \%-3 \%$ or even below the detection limit of EDX, showing no Mn-rich inclusions down to the resolution limit of EDX (about $1 \mu \mathrm{m}$ ). Mn-rich regions (dark areas in Fig. 1) which are smaller than Mn-poor regions show higher Mn concentration, with some regions having the composition close to $\mathrm{Mn}_{5} \mathrm{Ge}_{3}$ and $\mathrm{Mn}_{11} \mathrm{Ge}_{8}$ phases. We found that the $\mathrm{Mn}$ content in the Mn-poor phase was similar for all samples with different initial Mn content. In the following, we will distinguish between the single-crystalline homogeneous Mnpoor phase containing 1\%-3\% of Mn and the highly inhomogeneous Mn-rich phase.

The crystal structure of the Mn-poor phase was investigated by $\theta-2 \theta$ x-ray powder diffraction (XRD) as well as by Laue back reflection on single crystals. Figure 2 shows an XRD pattern of the Mn-poor region of the $\mathrm{Mn}_{0.08} \mathrm{Ge}_{0.92}$ sample indicating good crystalline quality of the samples without any impurity phases. The XRD pattern was that expected for the diamond structure of Ge. However, the Mnrich parts of the single crystals showed additional peaks which can be attributed to $\mathrm{Mn}_{5} \mathrm{Ge}_{3}$ and $\mathrm{Mn}_{11} \mathrm{Ge}_{8}$ phases (not shown). The Laue diffraction pattern measured in the [100] direction of the Mn-poor region of $\mathrm{Mn}_{0.08} \mathrm{Ge}_{0.92}$ sample is shown as an inset in Fig. 2. A fourfold symmetry was observed due to the [100] direction of the single crystal, confirming the single-crystal quality of the Mn-poor phase.

\section{B. Magnetic properties}

Magnetic properties of the samples were studied by means of superconducting quantum interference device (SQUID) magnetometry. For this purpose the single-crystal Mn-poor part was separated from the rest of the crystal. Figure 3(a) shows a typical temperature-dependent magnetization measured in an applied field of $10 \mathrm{mT}$ in zero-field cooled (ZFC) and field-cooled (FC) conditions. In the $M(T)$ curves two distinct transitions can be identified at about 150 and $285 \mathrm{~K}$. Only in the region between 150 and $285 \mathrm{~K}$ a hysteresis in the $M(H)$ dependence could be observed [see Fig. 4(a)]. Similar magnetic transitions in $\mathrm{Mn}_{x} \mathrm{Ge}_{1-x}$ single crystals were observed by Cho et al. ${ }^{5}$ and attributed to an antiferromagnetic state to ferromagnetic state transition at 

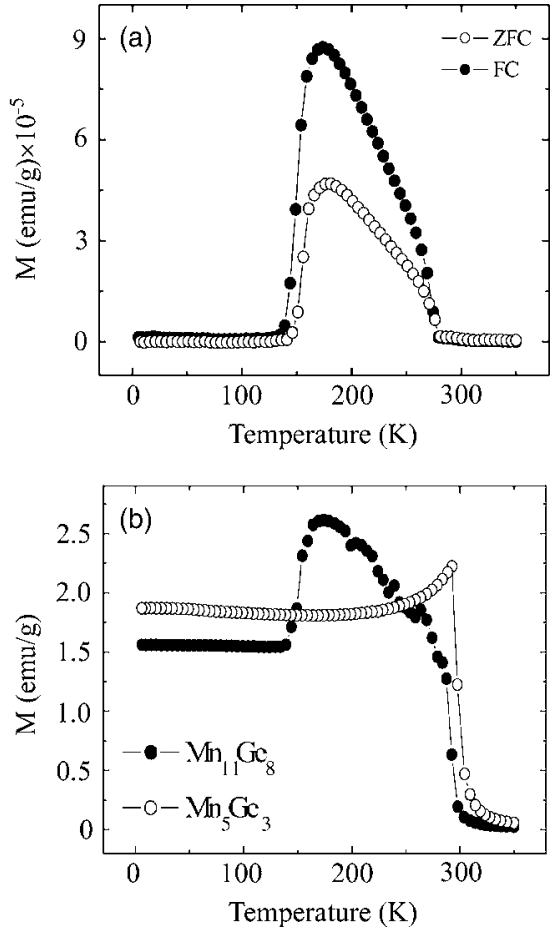

FIG. 3. (a) Temperature-dependent magnetization $M(T)$ of the Mn-poor part of a nominal $\mathrm{Mn}_{0.08} \mathrm{Ge}_{0.92}$ sample measured in a $10 \mathrm{mT}$ magnetic field under field cooled (solid circle) and zero-field cooled (open circle) conditions. In (b) temperature-dependent magnetization $M(T)$ measured in a $10 \mathrm{mT}$ magnetic field for field cooled $\mathrm{Mn}_{11} \mathrm{Ge}_{8}$ (solid circle) and $\mathrm{Mn}_{5} \mathrm{Ge}_{3}$ (open circles) reference samples is shown.

$150 \mathrm{~K}$ and an ferromagnetic state to paramagnetic state transition at $285 \mathrm{~K}$. On the other hand, recent investigations on epitaxial $\mathrm{Mn}_{x} \mathrm{Ge}_{1-x}$ thin films do not show comparable magnetic transitions, ${ }^{3,4}$ which was attributed to the absence of Mn-rich alloy phases in the sample.

In order to investigate the origin of this magnetic behavior, we compared the $M(T)$ data obtained from the $\mathrm{Mn}_{x} \mathrm{Ge}_{1-x}$ samples with the data from two reference samples: $\mathrm{Mn}_{11} \mathrm{Ge}_{8}$ and $\mathrm{Mn}_{5} \mathrm{Ge}_{3}$ [see Fig. 3(b)]. $\mathrm{Mn}_{11} \mathrm{Ge}_{8}$ and $\mathrm{Mn}_{5} \mathrm{Ge}_{3}$ were taken as references, because these compounds can be produced during single-crystal growth. ${ }^{13}$ In Fig. 3(b) $M(T)$ curves of both reference compounds $\left(\mathrm{Mn}_{5} \mathrm{Ge}_{3}\right.$ and $\left.\mathrm{Mn}_{11} \mathrm{Ge}_{8}\right)$ are presented. $\mathrm{Mn}_{5} \mathrm{Ge}_{3}$ has $T_{C}$ of about $300 \mathrm{~K},{ }^{14}$ whereas for $\mathrm{Mn}_{11} \mathrm{Ge}_{8}$ two transition temperatures at 150 and $285 \mathrm{~K}$ can be observed, indicating the existence of a ferromagnetic phase between 150 and $285 \mathrm{~K}$. In contrast to the findings by Yamada et al., ${ }^{15}$ the magnetization measured below $150 \mathrm{~K}$ does not decrease to zero for $\mathrm{Mn}_{11} \mathrm{Ge}_{8}$, indicating the presence of a ferromagnetic phase below $150 \mathrm{~K}$. The $\mathrm{Mn}_{11} \mathrm{Ge}_{8}$ sample used in the present study possibly contains a small amount of $\mathrm{Mn}_{5} \mathrm{Ge}_{3}$ responsible for ferromagnetism below $150 \mathrm{~K}$. The Mn-poor part of the nominal $\mathrm{Mn}_{0.08} \mathrm{Ge}_{0.92}$ sample exhibits two transition temperatures which correlate with the data from the polycrystalline $\mathrm{Mn}_{11} \mathrm{Ge}_{8}$ sample. However, no ferromagnetic state was observed in the $\mathrm{Mn}_{0.08} \mathrm{Ge}_{0.92}$ sample below $150 \mathrm{~K}$. Thus, the ferromagnetic phase found between 150 and $285 \mathrm{~K}$ in the $\mathrm{Mn}_{0.08} \mathrm{Ge}_{0.92}$ is probably due to the $\mathrm{Mn}_{11} \mathrm{Ge}_{8}$ phase incorporated into the Ge single crystal. Recent $M(T)$ measurements performed on the MBE-grown $\mathrm{Mn}_{x} \mathrm{Ge}_{1-x}$ samples also show a transition temperature at
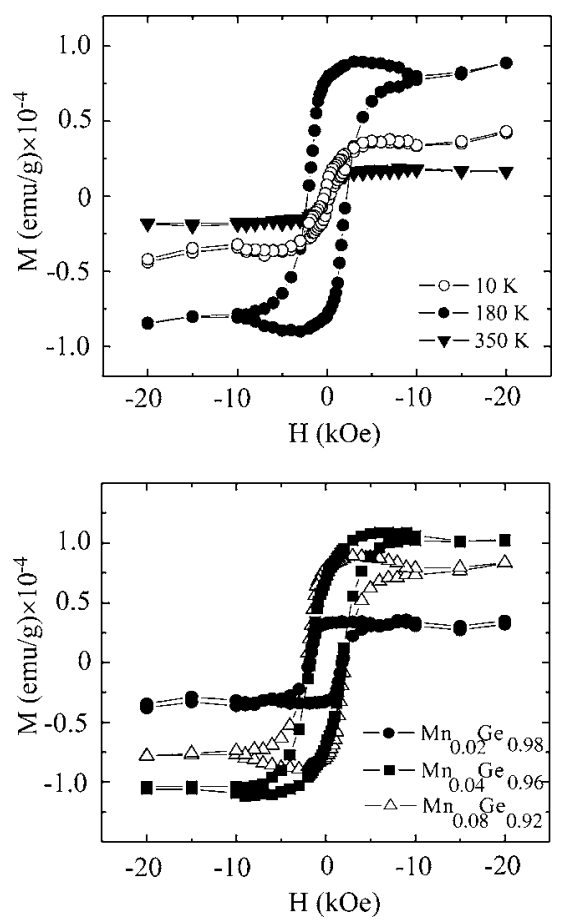

FIG. 4. (a) Magnetic hysteresis loops of the Mn-poor part of the nominal $\mathrm{Mn}_{0.08} \mathrm{Ge}_{0.92}$ sample recorded at $10 \mathrm{~K}$ (open circle), $180 \mathrm{~K}$ (solid circle), and $350 \mathrm{~K}$ (triangle down). Only at $180 \mathrm{~K}$ a distinctive loop is visible due to ferromagnetic behavior. In (b) magnetic hysteresis loops of Mn-poor parts of $\mathrm{Mn}_{0.02} \mathrm{Ge}_{0.98}$ (solid circle), $\mathrm{Mn}_{0.04} \mathrm{Ge}_{0.96}$ (solid square), and $\mathrm{Mn}_{0.08} \mathrm{Ge}_{0.92}$ (open triangle up) samples measured at $180 \mathrm{~K}$ are shown.

about $285 \mathrm{~K}$ which is due to the existence of a Mn-rich ordered phase $\left(\mathrm{Mn}_{5} \mathrm{Ge}_{3}\right)$ (Ref. 16) and is not an intrinsic property of the DMS. A small divergence of ZFC and FC measurements on the $\mathrm{Mn}_{0.08} \mathrm{Ge}_{0.92}$ sample is visible which is often attributed to a blocking transition of interacting superparamagnetic Mn-rich clusters of different size incorporated onto Ge matrix as shown by Park et al. ${ }^{17}$ Recent temperature-dependent magnetization measurements on MBE-grown $\mathrm{Mn}_{x} \mathrm{Ge}_{1-x}$ samples showed the presence of two different magnetic phases. ${ }^{18}$ Superparamagnetic $\mathrm{Mn}_{5} \mathrm{Ge}_{3}$ clusters were reported to be the origin of a blocking transition at high temperature around $210 \mathrm{~K}$, whereas superparamagnetic Mn-rich nanoclusters cause a blocking transition around $12 \mathrm{~K}$ that is attributed to spin-glass behavior.

Figure 4(a) displays magnetic hysteresis loops of the Mn-poor part of the nominal $\mathrm{Mn}_{0.08} \mathrm{Ge}_{0.92}$ sample measured at different temperatures. Clear ferromagnetic behavior is observed at $180 \mathrm{~K}$. At room temperature the coercive field $\left(H_{C}\right)$ decreases to zero which correlates with the magnetic phase transition at $285 \mathrm{~K}$ shown in Fig. 3(a). The saturation magnetization at $180 \mathrm{~K}$ is about $5-10 \times 10^{-5} \mathrm{emu} / \mathrm{g}$ and the coercive field is about $2 \mathrm{kOe}$ for all $\mathrm{Mn}_{x} \mathrm{Ge}_{1-x}$ samples [see Fig. 4(b)], showing that different dilution contents have not been achieved during sample preparation, which was also confirmed by EDX measurements. Comparable values of $H_{C}$ were observed by D'Orazio et al. ${ }^{19}$ in $\mathrm{Mn}$ ion implanted Ge and were attributed to the formation of Mn-rich particles. Moreover, $H_{C}$ of pure $\mathrm{Mn}_{5} \mathrm{Ge}_{3}$ (Ref. 20) was found to be in the range of the coercive field measured in our samples. 


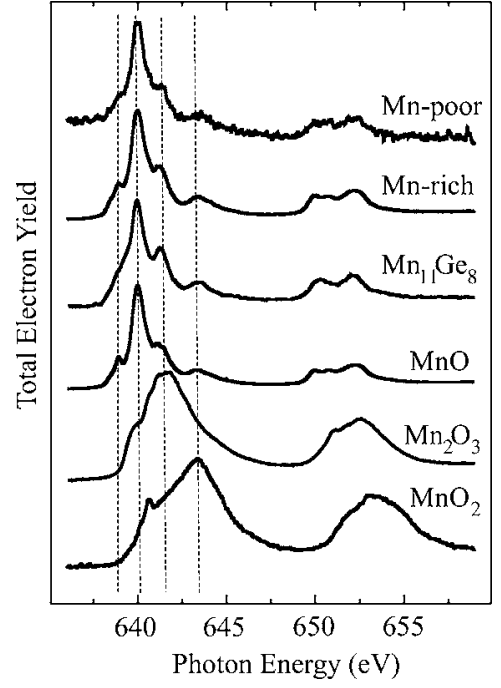

FIG. 5. Mn $L_{2,3}$ XAS spectra of the $\mathrm{Mn}_{0.1} \mathrm{Ge}_{0.9}$ sample obtained for a Mnrich and $\mathrm{Mn}$-poor part together with the reference spectra of $\mathrm{Mn}_{11} \mathrm{Ge}_{8}, \mathrm{MnO}$, $\mathrm{Mn}_{2} \mathrm{O}_{3}$, and $\mathrm{MnO}_{2}$.

Thus, ferromagnetic behavior in our samples can be attributed to the Mn-rich particles of a submicron size homogeneously distributed in the Ge matrix.

A linear increase of $T_{C}$ as a function of increasing $\mathrm{Mn}$ dilution content was shown experimentally. ${ }^{3,6}$ In the present $\mathrm{Mn}_{x} \mathrm{Ge}_{1-x}$ samples the transition temperature does not depend on the Mn-content of the samples; in fact, $T_{C}$ remains constant due to the presence of Mn-rich phase $\left(\mathrm{Mn}_{11} \mathrm{Ge}_{8}\right)$.

\section{Electronic properties}

In order to investigate the electronic structure of the nominal $\mathrm{Mn}_{0.1} \mathrm{Ge}_{0.9}$ sample, Mn $L_{2,3}$ XAS measurements were performed for Mn-rich and Mn-poor areas of the $\mathrm{Mn}_{0.1} \mathrm{Ge}_{0.9}$ sample. Figure 5 shows $\mathrm{Mn} L_{2,3}$ XAS spectra obtained from the Mn-rich as well as the Mn-poor regions, together with the reference absorption spectra of $\mathrm{Mn}_{11} \mathrm{Ge}_{8}$, $\mathrm{MnO}\left(\mathrm{Mn}^{2+}\right), \mathrm{Mn}_{2} \mathrm{O}_{3}\left(\mathrm{Mn}^{3+}\right)$, and $\mathrm{MnO}_{2}\left(\mathrm{Mn}^{4+}\right)$. The $\mathrm{Mn}$ $L_{2,3}$ XAS spectra of the Mn-rich and Mn-poor parts of the $\mathrm{Mn}_{0.1} \mathrm{Ge}_{0.9}$ sample show similar line shapes which are close to that of $\mathrm{MnO}$ but quite different from $\mathrm{Mn}_{2} \mathrm{O}_{3}$ and $\mathrm{MnO}_{2}$, suggesting that most of the $\mathrm{Mn}$ is present in the $\mathrm{Mn}^{2+}$ state. Thus, the existence of metallic Mn in the Mn-rich as well as in the Mn-poor parts of the $\mathrm{Mn}_{0.1} \mathrm{Ge}_{0.9}$ sample can be ruled out. The XAS spectra of the Mn-poor part showed a lower intensity compared with that of the Mn-rich part, confirming a lower Mn concentration (the XAS spectra in Fig. 5 were normalized to the maximum intensity). A comparable $\mathrm{Mn}$ $L_{2,3}$ XAS line shape was reported by Kang et al. ${ }^{12}$ measured on the inhomogeneous DMS $\mathrm{Mn}_{x} \mathrm{Ge}_{1-x}$. The spectra are also found to be in good agreement with the XAS spectrum calculated for the tetrahedrally coordinated $\mathrm{Mn}^{2+}$ in the highspin $(S=5 / 2)$ configuration. ${ }^{12}$

Figure 6 shows valence band photoelectron spectroscopy of the Mn-poor part of the $\mathrm{Mn}_{0.1} \mathrm{Ge}_{0.9}$ sample performed around the Mn $2 p_{3 / 2}$ absorption edge. The off-resonance spectrum $(h \nu=634 \mathrm{eV})$ shows a line shape similar to that of pure Ge which is expected from the small Mn concentration. The spectral line shape of the on-resonance spectra $(h \nu$

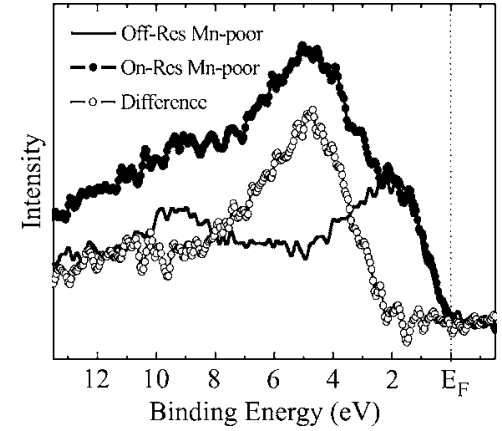

FIG. 6. Resonant photoemission spectroscopy of the Mn-poor part of the $\mathrm{Mn}_{0.1} \mathrm{Ge}_{0.9}$ sample around the $\mathrm{Mn} 2 p_{3 / 2}$ absorption edge. On-resonance $(h \nu=640 \mathrm{eV})$ and off-resonance $(h \nu=634 \mathrm{eV})$ valence band spectra together with the difference curve (open circle) reflecting the Mn $3 d$ PDOS are shown.

$=640 \mathrm{eV})$ measured for the Mn-poor part of the $\mathrm{Mn}_{x} \mathrm{Ge}_{1-x}$ samples shows a broad feature around $4.8 \mathrm{eV}$, which is in agreement with previous studies on phase-separated $\mathrm{Mn}_{x} \mathrm{Ge}_{1-x}{ }^{12,21}$ The difference between the on-resonance and off-resonance spectra is proportional to the Mn $3 d$ PDOS. The extracted Mn $3 d$ PDOS exhibits a peak centered at 4.8 $\mathrm{eV}$ and a weak tail up to $11 \mathrm{eV}$. The Mn $3 d$ PDOS shows that $\mathrm{Mn} 3 d$ states are located well below $\mathrm{E}_{F}$, indicating that $\mathrm{Mn}$ $3 d$ electrons occupy the deep levels. The peak in Mn $3 d$ PDOS centered at $4.8 \mathrm{eV}$ is in good agreement with a similar feature of the $\mathrm{Mn}_{5} \mathrm{Ge}_{3}$ phase ${ }^{21}$ as well as of the ion implanted $\mathrm{Mn}_{x} \mathrm{Ge}_{1-x}$ samples containing $\mathrm{Mn}_{5} \mathrm{Ge}_{3}$ nanoparticles. ${ }^{22}$ Moreover, the spectral features related to the effective dilution of substitutional $\mathrm{Mn}$ in the Ge are expected to appear at about $4 \mathrm{eV}, 22$ which is not in agreement with our measurements. Thus, the present PES measurements give a strong indication that even in the Mn-poor part of the $\mathrm{Mn}_{x} \mathrm{Ge}_{1-x}$ sample $\mathrm{Mn}$ is present in the form of Mn-rich phase $\mathrm{Mn}_{5} \mathrm{Ge}_{3}$.

\section{CONCLUSION}

In conclusion, we investigated structural, magnetic, and electronic properties of $\mathrm{Mn}_{x} \mathrm{Ge}_{1-x}$ samples grown by the Bridgman's crystal growth technique. EDX used for the investigation of the chemical distribution of $\mathrm{Mn}$ in Ge clearly shows a strong phase separation into the single-crystalline homogeneous Mn-poor phase containing 1\%-3\% of Mn and the highly inhomogeneous Mn-rich phase containing the regions having the composition close to $\mathrm{Mn}_{5} \mathrm{Ge}_{3}$ and $\mathrm{Mn}_{11} \mathrm{Ge}_{8}$ phases. Temperature-dependent magnetization measurements on the Mn-poor part of the $\mathrm{Mn}_{x} \mathrm{Ge}_{1-x}$ samples show ferromagnetic transitions at 150 and $285 \mathrm{~K}$ which correspond to those of the $\mathrm{Mn}_{11} \mathrm{Ge}_{8}$ reference sample. The observed magnetic behavior in both parts of the sample can be attributed to the Mn-rich submicron-sized clusters homogeneously distributed in the Ge matrix. Mn $L_{2,3}$ XAS measurements show that $\mathrm{Mn}$ ions in the Mn-rich and Mn-poor parts are present in the divalent high-spin state. The presence of the Mn-rich phases in the Mn-poor parts was also demonstrated with ResPES measurements performed at the Mn $2 p_{3 / 2}$ absorption edge. The obtained Mn $3 d$ PDOS shows a feature centered at $4.8 \mathrm{eV}$ which is characteristic for the $\mathrm{Mn}_{5} \mathrm{Ge}_{3}$ phase. In this study we demonstrate that ferromagnetism observed in both 
parts of the $\mathrm{Mn}_{x} \mathrm{Ge}_{1-x}$ samples is due to the Mn-rich clusters in the strongly phase-separated $\mathrm{Mn}_{x} \mathrm{Ge}_{1-x}$, indicating that the single-crystal growth technique is not an appropriate method for the DMS preparation.

\section{ACKNOWLEDGMENTS}

We would like to thank U. Probst for fruitful discussions. E.B. and M.F. acknowledge the financial support by BESSY (Berlin). Part of this work was supported by SFB 513.

${ }^{1}$ H. Ohno, Science 281, 951 (1998).

${ }^{2}$ T. Dietl, H. Ohno, F. Matsukura, J. Cibert, and D. Ferrand, Science 287 1019 (2000).

${ }^{3}$ Y. D. Park, A. T. Hanbicki, S. C. Erwin, C. S. Hellberg, J. M. Sullivan, J. E. Mattson, T. F. Ambrose, A. Wilson, G. Spanos, and B. T. Jonker, Science 295, 651 (2002).

${ }^{4}$ F. D‘Orazio, F. Lucari, N. Pinto, L. Morresi, and R. Murri, J. Magn. Magn. Mater. 272-276, 2006 (2004).

${ }^{5}$ S. Cho, S. Choi, S. C. Hong, Y. Kim, J. B. Ketterson, B.-J. Kim, Y. C. Kim, and J.-H. Jung, Phys. Rev. B 66, 033303 (2002).

${ }^{6}$ F. Tsui, L. He, L. Ma, A. Tkachuk, Y. S. Chu, K. Nakajima, and T. Chikyow, Phys. Rev. Lett. 91, 177203 (2003).

${ }^{7}$ A. P. Li, J. F. Wendelken, J. Shen, L. C. Feldman, J. R. Thompson, and H. H. Weitering, Phys. Rev. B 72, 195205 (2005).

${ }^{8}$ D. Bougeard, S. Ahlers, A. Trampert, N. Sircar, and G. Abstreiter, Phys. Rev. Lett. 97, 237202 (2006).
${ }^{9}$ S. Ahlers, D. Bougeard, N. Sircar, G. Abstreiter, A. Trampert, M. Opel, and R. Gross, Phys. Rev. B 74, 214411 (2006).

${ }^{10}$ L. Lifeng, C. Nuofu, C. Chenlong, L. Yanli, Y. Zhigang, and Y. Fei, J. Cryst. Growth 273, 106 (2004).

${ }^{11}$ S. Picozzi, L. Ottaviano, M. Passacantando, G. Profeta, A. Continenza, F. Priolo, M. Kim, and A. J. Freeman, Appl. Phys. Lett. 86, 062501 (2005).

${ }^{12}$ J.-S. Kang, G. Kim, S. C. Wi, S. S. Lee, S. Choi, S. Cho, S. W. Han, K. H. Kim, H. J. Song, H. J. Shin, A. Sekiyama, S. Kasai, S. Suga, and B. I. Min, Phys. Rev. Lett. 94, 147202 (2005).

${ }^{13}$ Binary Alloy Phase Diagrams, 2nd ed., edited by T. B. Massalski (ASM International, Materials Park, OH, 1990).

${ }^{14}$ X. B. Liu and Z. Altounian, J. Appl. Phys., 99, 08 Q101 (2006).

${ }^{15}$ N. Yamada, K. Maeda, Y. Usami, and T. Ohoyama, J. Phys. Soc. Jpn. 55, 3721 (1986).

${ }^{16}$ C. Bihler, C. Jaeger, T. Vallaitis, M. Gjukic, M. S. Brandt, E. Pippel, J. Woltersdorf, and U. Gösele, Appl. Phys. Lett. 88, 112506 (2006).

${ }^{17}$ Y. D. Park, A. Wilson, A. T. Hanbicki, J. E. Mattson, T. Ambrose, G. Spanos, and B. T. Jonker, Appl. Phys. Lett. 78, 2739 (2001).

${ }^{18}$ C. Jaeger, C. Bihler, T. Vallaitis, S. T. B. Goennenwein, M. Opel, R. Gross, and M. S. Brandt, Phys. Rev. B 74, 045330 (2006).

${ }^{19}$ F. D‘Orazio, F. Lucari, M. Passacantando, P. Picozzi, S. Santucci, and A. Verna, IEEE Trans. Magn. 38, 2856 (2002).

${ }^{20}$ C. Zeng, S. C. Erwin, L. C. Feldman, A. P. Li, R. Jin, Y. Song, J. R. Thompson, and H. H. Weitering, Appl. Phys. Lett. 83, 5002 (2003).

${ }^{21}$ C. Zeng, W. Zhu, S. C. Erwin, Z. Zhang, and H. H. Weitering, Phys. Rev. B 70, 205340 (2004)

${ }^{22}$ L. Ottaviano, M. Passacantando, S. Picozzi, A. Continenza, R. Gunnella, A. Verna, G. Bihlmayer, G. Impellizzeri, and F. Priolo, Appl. Phys. Lett. 88, 061907 (2006). 